\title{
Delayed prescribing for upper respiratory tract infections: a qualitative study of GPs' views and experiences
}

\author{
Sigurd Høye, Jan C Frich and Morten Lindbok
}

\begin{abstract}
Background

Delayed prescribing has been promoted as a strategy that meets patients' expectations and helps to avoid unnecessary use of antibiotics in upper respiratory tract infections.

Aim

To explore GPs' views on and experiences with delayed prescribing in patients with acute upper respiratory tract infections.

\section{Design of study}

Qualitative study involving focus groups.

\section{Setting}

Norwegian general practice.

\section{Method}

Qualitative analysis of data collected from five focus groups comprising 33 GPs who took part in a qualityimprovement programme of antibiotic prescribing.

\section{Results}

The views of GPs differed on the usefulness of delayed prescribing. GPs who endorsed the strategy emphasised shared decision making and the creation of opportunities for educating patients, whereas GPs who were negative applied the strategy mainly when being pressed to prescribe. Mild and mainly harmless conditions of a possible bacterial origin, such as acute sinusitis and acute otitis, were considered most suitable for delayed prescribing. A key argument for issuing a wait-and-see prescription was that it helped patients avoid seeking after-hours care. For issuing a wait-and-see prescription, the GPs required that the patient was 'knowledgeable', able to understand the indications for antibiotics, and motivated for shared decision making. GPs emphasised that patients should be informed thoroughly when receiving a wait-and-see prescription.

\section{Conclusion}

Not all GPs endorse delayed prescribing; however, it appears to be a feasible approach for managing patients with early symptoms of mild upper respiratory tract infections of a possible bacterial origin. Informing the patients properly while issuing wait-and-see prescriptions is essential.
\end{abstract}

\section{Keywords}

antibacterial agents; drug prescriptions; family practice; qualitative research; upper respiratory tract infections.

\section{INTRODUCTION}

More than $90 \%$ of antibiotic prescriptions in Norway are issued by GPs, and about $60 \%$ of these are prescribed for common respiratory tract infections. ${ }^{1}$ Conditions that most frequently lead to antibiotic prescriptions are acute bronchitis, acute upper respiratory tract infections (URTIs), and acute otitis. ${ }^{1}$ Antibiotics have only a modest role in self-limiting URTIs. ${ }^{2-4}$ Overuse of antibiotics may result in adverse drug reactions, ${ }^{4}$ development of resistant microbes, ${ }^{5}$ and medicalisation of self-limiting disease. ${ }^{6}$

Delayed prescribing has been advocated as a strategy that meets patients' expectations and, at the same time, helps avoid unnecessary use of antibiotics. ${ }^{7,8}$ The method is used in either of the following two ways: GPs can provide a 'wait-and-see prescription' and advise the patient to wait until using the prescription, hoping that the symptoms resolve, ${ }^{9}$ or GPs can ask the patient to come back for a prescription if symptoms persist or worsen. ${ }^{10}$ Several randomised controlled trials have been carried out to investigate the effectiveness of waitand-see prescriptions, regarding the ability to decrease antibiotic consumption, and the safety of the method. Reported pick-up rates for antibiotics vary $-24-38 \%$ (otitis media), ${ }^{9,11} 31 \%$ (sore throat), ${ }^{10}$

$S$ Høye, MD, researcher; $M$ Lindboek, $M D, P h D$, professor of general practice, Antibiotic Center for Primary Care, Department of General Practice and Community Medicine; JC Frich, MD, PhD, associate professor of health management, Department of Health Management and Health Economics, Institute of Health and Society, University of Oslo, Norway.

Address for correspondence

Dr Sigurd Høye, Antibiotic Center for Primary Care, Department of General Practice and Community Medicine, Institute of Health and Society, University of Oslo, PO Box 1130 Blindern, N-0318 Oslo, Norway. E-mail: sigurd.hoye@medisin.uio.no

Submitted: 30 November 2009; Editor's response: 3 March 2010; final acceptance: 13 April 2010.

(๑) British Journal of General Practice 2010; 60: 907-912. 


\section{How this fits in}

Antibiotics have only a modest role in the treatment of self-limiting upper respiratory tract infections and inappropriate prescription of antibiotics is common in general practice. Delayed prescribing is a tool that can help reduce unnecessary use of antibiotics; GPs prefer to use delayed prescribing in conditions where antibiotics may be indicated. GPs may find the strategy useful even if they do not experience exaggerated expectations from the patient.

$20-45 \%$ (cough), ${ }^{12,13}$ and $48 \%$ (common cold) ${ }^{14}-$ with no difference in complication rates between patients given delayed and immediate antibiotics.

According to the findings from a Cochrane Review, ${ }^{15}$ immediate prescription was most likely to provide the best clinical outcome in patients with sore throat and otitis media, and delaying or avoiding antibiotics reduced antibiotic use for acute respiratory tract infections. The authors argue that delaying antibiotics has little advantage over avoiding them altogether when it is safe to do so. Still, doctors prescribe antibiotics when it is not necessary and when it is safe to avoid them. ${ }^{16}$ This could be because the prescribing is influenced by the doctor's impression of patient expectations. ${ }^{17,18}$ Outpatient antibiotic use is relatively low in Norway; that is, at the same level as other Northern European countries..$^{3,5}$ In addition, research suggests that Norwegian patients do not necessarily expect antibiotics for the treatment of URTIs. ${ }^{18}$

Little is known about Norwegian GPs' views and experiences of delayed prescribing. The study's authors' preconception was that GPs recognised delayed prescribing as a tool for reducing unnecessary antibiotic prescribing in patients with URTIs. They had an interest in whether GPs in a northern European setting, characterised with low prescription rates and low patient expectation for antibiotics, would consider delayed prescribing a feasible strategy. This study sought to examine the potentials and pitfalls with delayed prescribing as

Table 1. Characteristics of the participating GPs, Rx-PAD study participants, and a Norwegian reference GP population.

\begin{tabular}{lccc} 
& $n$ & $\begin{array}{c}\text { Mean age, } \\
\text { years (range) }\end{array}$ & $\begin{array}{c}\text { Mean antibiotic prescription } \\
\text { rate (range) }\end{array}$ \\
\hline Participating GPs & 30 & $50(33-67)$ & $388(151-635)$ \\
\hline Female & 14 & $47(35-64)$ & $361(151-508)$ \\
\hline Male & 16 & $52(33-67)$ & $412(229-635)$ \\
\hline Rx-PAD study GPs & 386 & $49(28-67)$ & $366(31-806)$ \\
\hline Vestfold study GPs ${ }^{21, b}$ & 145 & $49(29-67)$ & $284(0-695)$ \\
\hline
\end{tabular}

aPatients receiving an antibiotic prescription per 1000 patients consulting with a respiratory tract infection. Data were available for 30 of 33 participating GPs. ${ }^{b}$ Data on dispensed antibiotics from 145 out of 152 GPs in Vestfold county during two winter months 2003. Rx$P A D=$ Prescription Peer Academic Detailing experienced by GPs and whether the strategy is suitable for all patients. This study was carried out to explore Norwegian GPs' views and experiences of delayed antibiotic prescribing in patients with URTIs to gain more knowledge of the feasibility of the strategy.

The terms 'delayed prescribing' and 'wait-and-see prescription' are both used in the literature. 'Waitand-see prescription' corresponds to the Norwegian expression used in this study. 'Delayed prescribing' is used for the strategy, and 'wait-and-see prescription' for the prescription itself.

\section{METHOD}

\section{Setting}

This study was conducted as a part of the Prescription Peer Academic Detailing (Rx-PAD) study, ${ }^{19}$ a cluster-randomised educational intervention in general practice, with the aim of improving antibiotic prescribing in respiratory tract infections. The participating GPs were counselled by trained colleagues in their continuous medical education (CME) groups, and guidelines were presented to the GPs in a 1-day seminar. One of the authors held a discussion on the evidence for delayed prescribing.

\section{Participants}

Between October 2006 and September 2007, five focus groups were conducted, comprising 33 GPs who participated in the Rx-PAD study. ${ }^{19}$ Focus group interviews were considered an appropriate method to highlight views and divergent opinions. ${ }^{20}$ The participant GPs and focus groups were recruited using a purposeful sampling strategy, and the study aimed for variety in the sample with respect to geography, age, sex, and antibiotic prescribing rates. Table 1 presents the characteristics of the focus groups participants and the entire Rx-PAD study participants. For comparison, the characteristics of a reference GP population of Vestfold county 21 is presented.

\section{Interviews}

The first author acted as moderator for four focus group interviews, while the second author was the moderator for the fifth interview. Moderators used an interview guide that was developed by the authors, and which covered experiences and views of the GPs on delayed prescribing. Each of the focus group interviews lasted for 45-75 minutes. GPs were encouraged to share their views in an open discussion. All discussions were audiorecorded digitally. Preliminary findings and questions from the first interviews were fed back into later focus groups for further discussion. 


\section{Analysis}

Audiorecordings were transcribed verbatim. The material was analysed using a procedure for content analysis referred to as 'systematic text condensations', 22,23 comprising the following four steps: (i) reading all the material to obtain an overal impression, and bracketing previous ideas and preconceptions; (ii) identifying units of meaning, representing different aspects of GPs' experiences with delayed prescribing, and coding for these; (iii) condensing and summarising the contents of each of the coded groups; and (iv) generalising descriptions and concepts about specific themes. All the authors took part in the analysis, and met to discuss the meaning of data and the interpretation of the material. NVivo7 software was used for coding. Quotes from interviews were translated from Norwegian to English by the authors.

\section{RESULTS}

\section{Delayed prescribing as a concept}

GPs used the term 'wait-and-see' both for scheduling a follow-up visit when needed and for the delayed prescribing approach. They described general practice as a branch of medicine in which it is common and often appropriate to wait and see:

'[Intern doctors in primary care] think too much hospital, they want to solve [the problem] here and now and they don't use the approach that we are used to in general practice, to wait and see.' (focus group [FG] 3)

General practice was characterised as a field where practical experiences stimulate the development of new methods, such as the use of wait-and-see prescription. None of the GPs had been educated in the use of delayed prescribing before entering the $\mathrm{Rx}$ PAD study, but several of them said that they had been using the method for years:

'It is within the spirit of general practice to start using a method that you think seems logical and correct ... without having a scientific reason to do it. That is the GP's best quality. They make something smart out of it.' (FG1)

The GPs considered themselves prudent prescribers, and they were well aware of the relatively low antibiotic prescription rates in Norway. Avoiding unnecessary use of antibiotics was seen as an important task, although they usually described delayed prescribing as a practical solution at the individual patient level.

One of the focus groups displayed mainly negative attitudes towards delayed prescribing.
Members of this group argued that there were few situations where they used the strategy, and this was mainly when patients demanded antibiotics. One GP said:

'I have used [wait-and-see prescriptions] for several years, but to a small extent only ... It is for those who want medication, though you argue that they don't need it, but then they win at the end and I say: "Can't you at least wait for a couple of days and see how it develops?" You become somewhat pragmatic with the years.' (FG3)

The other focus groups, however, were positive about delayed prescribing. They found the method useful in several situations where the question of whether to prescribe was not clear. They also reported that they did not experience delayed prescribing solely as a compromise:

Moderator (M): 'Some have said that [delayed prescribing] can be a negotiated solution, do you have that experience?'

GP1: 'No.'

GP2: 'No. Who should negotiate?'

M: 'The patient wants [antibiotics], the doctor doesn't want to give [antibiotics].'

GP2: 'That was 20 years ago.' (FG4)

\section{Medical conditions}

GPs conveyed different views about what conditions they considered the most suitable for delayed prescribing. A widely held view was that delayed prescribing was appropriate in cases where GPs expected that the indication for antibiotics would be established within a few days. If they thought that additional observation would not add more information about whether to prescribe antibiotics, they would rather make that decision at the patient's first visit.

Some GPs argued that the wait-and-see strategy was feasible in cases where they were certain that the infection was caused by bacteria, but where the symptoms were mild; for example, a sore throat caused by streptococcal infection. GPs who held this view emphasised that in such cases, they knew antibiotics would be effective if needed. This view was opposed by other GPs who argued that infections with unknown aetiology were the ones most suitable for delayed prescribing.

Acute sinusitis was found to be the most suitable diagnosis for delayed prescribing. GPs considered bacterial sinusitis to be difficult to diagnose, and hence this was used as argument for delayed prescribing. One GP said: 
'[T]here are incredible few objective findings in sinusitis [...] a matter of opinion and assessment of symptoms is used to make decisions about treatment, and in such cases I think I might use wait-and-see.' (FG3)

Many GPs spoke about a certain group of patients with recurrent sinus symptoms, who consulted them too early during the course of illness and made demands for antibiotics. In such cases, the GPs could advise the patients to use non-antibiotic treatment options for sinus symptoms, such as decongestants and analgesics, as an alternative to prescribing antibiotics at the patient's first visit.

Children with acute otitis media were also seen as suitable for delayed prescribing. GPs reported that they found it easier to avoid prescription of antibiotics to children at present than several years ago, because parents had become more sceptical about antibiotics.

A third diagnosis that could be appropriate for delayed prescribing was acute bronchitis in situations where point-of-care tests could not clearly separate viral bronchitis from pneumonia.

\section{Situational characteristics}

The GPs said that they more often issued a wait-andsee prescription if a weekend or holiday was approaching; this was reflected in the notion of 'Friday prescriptions'. It was important for the GPs to help their patients so that they did not have to seek after-hours care, because of the long distances and long waiting time at the after-hours care facility. In addition, the GPs reported that the after-hours care doctor might initiate treatment that they did not agree with, such as prescribing broad-spectrum antibiotics for harmless infections. The GPs, therefore, preferred to prescribe narrow-spectrum penicillin as a waitand-see prescription prior to the weekend. Following this strategy also contributed to reducing the workload of the after-hours care doctors.

The GPs would more easily issue a wait-and-see prescription in their regular list patient practice than in the after-hours care setting, because the scope for follow-up was better in the regular office setting, and also because the GPs normally knew their own patients better than the after-hours care patients.

\section{Characteristics of the patients}

There was agreement among the GPs that delayed prescribing did not suit all patients. Patients considered eligible for delayed prescribing were said to possess characteristics such as being 'knowledgeable' and 'bright'. The reason behind this thought was that patients with these characteristics would not violate the principle of wait-and-see and 'misuse' the prescription, and also to ensure that patients were able to evaluate their condition and make the right decision after the waiting period. One GP said:

'The patient has to understand the message and has to participate in the dialogue, and that it is wise to wait a bit and see. Otherwise, if I prescribe a wait-and-see prescription to a patient who is not following me in a way, I do not think that he waits and sees at all, and then I resign instead.' (FG1)

A patient was ascertained to be 'knowledgeable' if he/she agreed with the view of the GP that antibiotics should not be used unnecessarily, and if the patient knew the difference between viruses and bacteria.

The GPs said that patients' expectation for antibiotics influenced their prescribing, and might lead them to prescribe antibiotics despite a lack of a medical indication. Issuing a wait-and-see prescription could be appropriate in such cases; the GPs could at least ask the patient to wait a few days and maybe keep them from unnecessary antibiotic treatment. Often the GPs described a wait-and-see prescription in this situation in negative terms: they had 'lost' the argument and chose a pragmatic solution, rather than an ideal one. There were also patients who did not want to decide on the use of antibiotics themselves, as discussed by two GPs:

'[It is] someone whom I know fairly well so that I know that it is not necessary to bring up the issue [wait-and-see prescription] at all. Yes, they want a clear answer. Either yes or no.' (FG1)

GPs agreed that one should not suggest issuing wait-and-see prescriptions to patients who were unwilling or incapable of making a decision on whether to use antibiotics.

\section{Informing the patient}

The GPs emphasised that informing the patient was mandatory when issuing a wait-and-see prescription. Two main themes were identified regarding this information. First, the patient should receive thorough information and clear instructions about when to start the medication. This was to ensure that the patient understood that there was a proper judgement behind the use of delayed prescribing, and to prevent patients from demanding a wait-andsee prescription for any respiratory tract infection. One GP said:

'If we just deliver a prescription and say "Start with the medication if you're not better in 
4 days", without giving more information about the condition and explain a little, then I think [the strategy] might have a slippery-slope effect. There needs to be a conversation to prevent this from happening.' (FG3)

Second, many of the GPs, particularly those who were positive towards delayed prescribing, considered wait-and-see prescription as a 'golden moment' for educating and empowering the patient.

\section{DISCUSSION}

\section{Summary of main findings}

The study found that GPs viewed delayed prescribing as a method that was well suited to the spirit of clinical work in general practice, although their views differed on the usefulness of the strategy. The GPs who endorsed delayed prescribing emphasised shared decision making and the opportunity to educate the patient. GPs who were negative said that they mainly used the strategy in what they experienced as an uncomfortable situation of being forced to prescribe.

Mild and mainly harmless conditions that might be of bacterial origin, such as acute sinusitis and acute otitis, were considered most suitable for delayed prescribing, as opposed to conditions that were considered as purely viral, such as common cold. An important argument for issuing a wait-and-see prescription was to help the patient to avoid seeking after-hours care, especially during holidays and weekends.

For issuing a wait-and-see prescription, the GPs required that the patient was 'knowledgeable', able to understand the indications for antibiotics, and motivated for shared decision making. GPs emphasised the importance of informing the patient thoroughly when issuing a wait-and-see prescription.

\section{Strengths and limitations of the study}

Participating GPs had agreed to take part in the RxPAD study, which aimed to improve antibiotic prescribing. Therefore, the GPs may have exhibited a positive view towards methods that aim to lower the use of antibiotics. However, both high and low prescribers are included in the focus groups, and the antibiotic prescription rates of the participants are somewhat higher than those of a reference GP population. ${ }^{21}$

GPs were educated on the use of delayed prescribing about 1 year before the focus group sessions. This might have influenced the participants' views. Although the representativeness may be questioned, the study findings reflect a diversity of views and experiences associated with delayed prescribing, and the authors believe that these findings are valid for Norwegian GPs practising delayed prescribing.

\section{Comparison with existing literature}

Delayed prescribing was not endorsed by all GPs in the study. This is in accordance with Arroll et $a l^{8}$ and Kumar et $a /,{ }^{24}$ who both found diverging views among GPs on the usefulness of the method.

In the literature, delayed prescribing is often referred to as a strategy for meeting the expectations of patients in situations where antibiotics are not indicated. ${ }^{7,8,15}$ In a study from New Zealand, Arroll et $a^{8}$ found that the physicians' motivation for delayed prescribing was primarily to decrease unnecessary antibiotic use, and that the method strengthened physician-patient relationships by helping the physicians to cope with the pressure from patients expecting antibiotics even for the treatment of the common cold. In the present study, however, GPs viewed delayed prescribing primarily as a practical solution for meeting a patient's need, such as helping the patient to avoid seeking after-hours care if the condition worsened during a weekend, and not as a specific method for decreasing the unnecessary use of antibiotics. The common cold was not viewed as a relevant condition for offering a wait-and-see prescription.

The antibiotic prescription rate for URTIs is higher in New Zealand compared with Norway. ${ }^{25}$ GPs in the current study setting probably experienced less pressure from patients demanding antibiotics than the GPs in the New Zealand study. Delayed prescribing may thus be a feasible strategy in settings with both high and low patient expectations for antibiotics.

Although it can be claimed that delaying antibiotics has fewer advantages over avoiding them, ${ }^{15}$ most GPs in the present study reported indentifying numerous situations where they viewed delayed prescribing as a reasonable approach. According to the national guidelines for antibiotic use in primary health care in Norway, ${ }^{26}$ acute sinusitis and acute otitis media are the only two URTI diagnoses where symptom duration is one of the criteria for antibiotic treatment (7 days for sinusitis and 1-3 days for otitis media). These are also the two diagnoses that the GPs found most suitable for delayed prescribing. It can be concluded that delayed prescribing could be used as a tool to follow treatment guidelines in URTIs.

\section{Implications for future research and clinical practice}

Delayed prescribing is not endorsed by all GPs, but appears to be a feasible approach among informed patients with early symptoms of mild URTIs of a 
possible bacterial origin, such as acute sinusitis. It is critically important that GPs keep their patients informed properly when they decide to put them on a wait-and-see prescription. Delayed prescribing has the potential to reduce unnecessary use of antibiotics in sinusitis, ${ }^{27}$ but further research is required to explore the effect of delayed antibiotics on the clinical outcome of sinusitis.

\section{Funding body}

This study was funded by a research scholarship from The General Practice Research Fund, The Norwegian Medical Association.

\section{Ethical approval}

The Rx-PAD study has been approved by The Regional Committee for Research Ethics. No additional approvement has been considered necessary for the present study, as no patients are involved.

\section{Competing interests}

The authors have stated that there are none.

\section{Acknowledgements}

We would like to thank the GPs who participated in this study.

\section{Discuss this article}

Contribute and read comments about this article on the Discussion Forum: http://www.rcgp.org.uk/bjgp-discuss

\section{REFERENCES}

1. Straand J, Rokstad KS, Sandvik H. Prescribing systemic antibiotics in general practice. A report from the More \& Romsdal Prescription Study. Scand J Prim Health Care 1998; 16(2): 121-127.

2. Arroll B. Antibiotics for upper respiratory tract infections: an overview of Cochrane reviews. Respir Med 2005; 99(3): 255-261.

3. Butler CC, Hood K, Verheij T, et al. Variation in antibiotic prescribing and its impact on recovery in patients with acute cough in primary care: prospective study in 13 countries. BMJ 2009; 338: b2242.

4. Butler CC, Rollnick S, Kinnersley P, et al. Reducing antibiotics for respiratory tract symptoms in primary care: consolidating 'why' and considering 'how'. Br J Gen Pract 1998; 48(437): 1865-1870.

5. Goossens H, Ferech M, Vander SR, Elseviers M. Outpatient antibiotic use in Europe and association with resistance: a cross-national database study. Lancet 2005; 365(9459): 579-587.

6. Little P, Gould C, Williamson I, et al. Reattendance and complications in a randomised trial of prescribing strategies for sore throat: the medicalising effect of prescribing antibiotics. BMJ 1997; 315(7104): 350-352.

7. Edwards M, Dennison J, Sedgwick P. Patients' responses to delayed antibiotic prescription for acute upper respiratory tract infections. $\mathrm{Br}$ $J$ Gen Pract 2003; 53(496): 845-850.

8. Arroll B, Goodyear-Smith F, Thomas DR, Kerse N. Delayed antibiotic prescriptions: what are the experiences and attitudes of physicians and patients? J Fam Pract 2002; 51(11): 954-959.
9. Spiro DM, Tay KY, Arnold DH, et al. Wait-and-see prescription for the treatment of acute otitis media: a randomized controlled trial. JAMA 2006; 296(10): 1235-1241.

10. Little P, Williamson I, Warner G, et al. Open randomised trial of prescribing strategies in managing sore throat. BMJ 1997; 314(7082): 722-727.

11. Little P, Gould C, Williamson I, et al. Pragmatic randomised controlled trial of two prescribing strategies for childhood acute otitis media. BMJ 2001; 322(7282): 336-342.

12. Dowell J, Pitkethly M, Bain J, Martin S. A randomised controlled trial of delayed antibiotic prescribing as a strategy for managing uncomplicated respiratory tract infection in primary care. $\mathrm{Br} \mathrm{J} \mathrm{Gen}$ Pract 2001; 51(464): 200-205.

13. Little P, Rumsby K, Kelly J, et al. Information leaflet and antibiotic prescribing strategies for acute lower respiratory tract infection: a randomized controlled trial. JAMA 2005; 293(24): 3029-3035.

14. Arroll B, Kenealy T, Kerse N. Do delayed prescriptions reduce the use of antibiotics for the common cold? A single-blind controlled trial. $J$ Fam Pract 2002; 51(4): 324-328.

15. Spurling GK, Del Mar CB, Dooley L, Foxlee R. Delayed antibiotics for respiratory infections. Cochrane Database Syst Rev 2007; (3): CD004417.

16. Petursson P. GPs' reasons for 'non-pharmacological' prescribing of antibiotics. A phenomenological study. Scand J Prim Health Care 2005; 23(2): 120-125.

17. Macfarlane J, Holmes W, Macfarlane R, Britten N. Influence of patients' expectations on antibiotic management of acute lower respiratory tract illness in general practice: questionnaire study. BM] 1997; 315(7117): 1211-1214.

18. Soma M, Slapgard H, Lerberg M, Lindbaek M. [Patients' expectations of antibiotics for acute respiratory tract infections]. Tidsskr Nor Laegeforen 2005; 125(15): 1994-1997.

19. Gjelstad S, Fetveit A, Straand J, et al. Can antibiotic prescriptions in respiratory tract infections be improved? A cluster-randomized educational intervention in general practice - the Prescription Peer Academic Detailing (Rx-PAD) Study [NCT00272155]. BMC Health Serv Res 2006; 6: 75.

20. Bloor M. Focus groups in social research. London: Sage Publications, 2001.

21. Gjelstad S, Dalen I, Lindbaek M. GPs' antibiotic prescription pattern for respiratory tract infections - still room for improvement. Scand J Prim Health Care 2009; 27(4): 208-215.

22. Giorgi A. Sketch of a psychological phenomenological method. In: Giorgi A (ed). Phenomenology and psychological research. Pittsburgh, PA: Duquesne University Press, 1985: 8-22.

23. Malterud K. Qualitative research: standards, challenges, and guidelines. Lancet 2001; 358(9280): 483-488.

24. Kumar S, Little P, Britten N. Why do general practitioners prescribe antibiotics for sore throat? Grounded theory interview study. BMJ 2003; 326(7381): 138.

25. McGregor A, Dovey S, Tilyard M. Antibiotic use in upper respiratory tract infections in New Zealand. Fam Pract 1995; 12(2): 166-170.

26. The Directorate of Health, The Antibiotic Center for Primary Health Care. National guidelines for antibiotic use in primary health care. Oslo: The Directorate of Health, 2008.

27. Martin CL, Njike VY, Katz DL. Back-up antibiotic prescriptions could reduce unnecessary antibiotic use in rhinosinusitis. J Clin Epidemiol 2004; 57(4): 429-434. 